\title{
ALE-BASED FSI SIMULATION OF SOLID PROPELLANT ROCKET INTERIOR
}

\author{
Sangho Han ${ }^{1}$ and Chongam Kim $^{1}$ \\ 1) Department of Aerospace Engineering, Seoul National University, Seoul 151-742, KOREA \\ Corresponding Author: Chongam Kim, Chongam@snu.ac.kr
}

\begin{abstract}
As a hybrid model of continuum motion description which combines the advantages of classical kinematical descriptions, i.e. Lagrangian and Eulerian description, the ALE (Arbitrary Lagrangian Eulerian) description is adopted for the simulation of a fluid-structure interaction of solid propellant rocket interior. The fluid-structure interaction process, combustion modeling, regression model and an automatic re-meshing algorithm are included to analyze an unsteady fluid-structure-combustion interaction phenomenon with the deformation of solid domain during the burning process. The developed solver is then applied for the ignition simulation of a solid propellant grain, which is a highly unsteady coupled phenomenon between gas flow and propellant structure. The computed results show complex flow physics in the combustion chamber and the behavior of a solid propellant deformation.
\end{abstract}

\section{INTRODUCTION}

The solid propellant rockets are in widely use for the reusable boosters of space launcher and military purposes and understanding the complex physics in a combustion chamber is extremely important for the development and maintenance of a solid propellant rocket. Among the large number of factor influencing the rocket interior physics, the main factors which govern the interior phenomena of the solid rocket are the flow of hot and high pressure gases, the deformation of the propellant grain and combustion process. Furthermore, each principal element (fluid, structure, combustion) governing complex physical phenomena makes a feedback cycle to influence one another. Thus, it is extremely complicated to accurately predict a deformed shape of a propellant grain during the combustion process.

The objective of this research is "Integrated analysis of Fluid-Structure-Combustion Interaction with momentum transfer and transient burning across a moving interface for solid propellant rocket system". In order to numerically analyze for this problem, we use following some methodologies. The Arbitrary Lagrangian-Eulerian method, Accurate and/or efficient solver for fluid, solid and combustion, data transfer scheme and FSI time marching scheme to integrate individual solver, and Automatic mesh generation and adaption method.

\section{SIMULATION METHODOLOGIES}

\section{ALE KINEMATICAL DESCRIPTION \\ The Arbitrary Lagrangian Eulerian (ALE) kinematical description [1] has been incorporated into fluid/solid formulation, to accurately capture the deformation of solid propellant grain. The ALE description is a hybrid approach which combines advantages of the classical Lagrangian and Eulerian formulations. In Lagrangian approach, mesh nodes are attached to continuum particles and move with them, while, in Eulerian approach, nodes of}


computational mesh remain fixed as continuum particles pass through them. So, it is clear that neither Lagrangian nor Eulerian approaches can properly describe the progress of solid propellant burning, because some particles are eroded due to the regression of the propellant surface. In the ALE approach, numerical simulations are firstly conducted in the Eulerian manner, and the deformation of computational mesh is described by the Lagrangian pattern. Thus the ALE approach is suitable for simulating the solid propellant rocket interior problem.

\section{FLUID COMPONENT}

We consider a two-dimensional axisymmetric compressible flow for the interior combustion chamber. Since the mass flux from the exposed grain boundary face will sweep away the boundary layer, we may neglect the viscous effect near the grain surface, and reasonable results can be obtained by the inviscid flow solver. But, at the early stage of the ignition process, complex flow physics influence the outbreak of the fire at the grain surface and ignition delay time. Besides, the vicinity of the slot, nozzle, and boots has complicated geometry, so viscous and turbulence effect cannot be disregarded in this region. Therefore, both inviscid and viscous flow simulation have been performed for rocket interior FSI simulation [2].

\section{SOLID COMPONENT}

Generally, the solid propellant grain exhibits visco-elastic characteristics. In this study, the mechanical response of the grain is simulated using the Arruda-Boyce nonlinear-elastic constitutive model, which has been shown to be quite successful in capturing the small and large strain response of filled elastomers, and the generalized Maxwell visco-elastic model, which has been developed to properly describe the energy absorbing behavior of dissipative materials, especially polymeric rubber materials.

\section{COMBUSTION COMPONENT}

Combustion is the important component for the solid rocket interior simulation. Generally, there are several factors which decide the burning rate of grain. One-dimensional transient burning model is used to efficiently simulate the burning process of an interface between fluid domain and propellant grain surface. This model can satisfy the Piobert's law, which defines the burnback characteristics propellant grain. We assume that the grain is heated by hot gas and ignited on its exposed inner surface when the grain surface temperature exceeds some specific value.

\section{SPATIAL AND TEMPORAL DATA TRANSFER SCHEME}

In this research, we adopted common refinement data transfer scheme as the 1-D case. But, in the 2-D case induced by re-meshing process, accuracy would be much more important than conservation. So in the 2-D case, cubic spline interpolation scheme would be more appropriate which may guarantee a reasonable accuracy with an acceptable computational cost. For spatial data transfer during the FSI simulation, Dirichlet velocity boundary condition and Neumann momentum boundary condition should be satisfied along fluid-solid interface.

$v_{f}=v_{s} ;$ Dirichlet condition,

$t_{f}=t_{s}$ where, $t_{f}=t_{s} \cdot n_{f}-\sigma_{f} \cdot n_{f}$ and $t_{s}=\sigma_{s} \cdot n_{s}$; Neumann condition.

At present, we obtained the FSI results using the conventional serial staggered scheme. 


\section{DYNAMIC MESH TREATMENT AND REGRESSION MODEL}

The mesh regeneration procedure is performed locally when the local geometric changes occur, and entirely when the global or excessive geometric changes take place so that the local handling cannot maintain mesh quality any longer [3]. During the rocket burning process, because of the existence of slot geometry, a lump of solid propellant grain should be separated into two lumps. And, solid propellant grain has two type of corner. One is convex type and the other is concave type corner. For precise shape tracking of solid propellant grain during the burning process, these two type of corner needs individualized handling which satisfy the Piobert's law. So, regression model, which reproduce and/or redistribute the solid propellant surface nodes, is developed.

\section{SIMULATION RESULTS}

Figure 1 shows a typical two-dimensional axisymmetric rocket geometry designed for the simulation purpose. The blue color indicates the fluid domain, the red color shows the deformable structure domain (the propellant grain) and the green color is the undeformable structural part composed of the rocket case, igniter and the nozzle.

Computational domain of the current simulation and initial burning process near the igniter are shown in Fig. 2. In Fig.2 (top), flow in the combustion chamber is developed by hot gas ejected from the igniter. This combustion gas heat the exposed grain surface especially near the trimmed slot surface, so the vicinity of trimmed slot ignites first and sends out the mass flux normal to the grain surface. The flame propagates very fast along the exposed grain surface, while the propagation is rather delayed inside the slot where initial cold air is congested. Finally, hot emission gas is filled throughout the chamber and whole grain surface produces mass flux.

During the combustion process, the propellant grain is burned away and deformed because of burning mechanism and a structural load from high pressure flow in the combustion chamber. Fig.2 (bottom) indicates the geometric change of a solid propellant grain at almost of contained propellant is consumed for propulsion.

\section{CONCLUSION}

This paper has presented an overview of the main components of a coupled fluidstructure-combustion algorithm developed to simulate the complex phenomena of the interior combustion chamber of a solid propellant rocket. Using the developed program, we have simulated the fluid-structure-combustion coupling phenomena. From the simulation results, we could observe the details of initial burning and flame propagation process of the exposed propellant grain surface and understand the structural deformation tendency of a solid propellant grain. In addition, while the existing experimental test could obtain only limited data of the flow fields, we could observe the variations of all flow variables at all points of the combustion chamber and structural information (displacement, stress, and so on) of the solid propellant grain and case.

\section{ACKNOWLEDGMENTS}

Authors appreciate the financial supports provided by NSL (National Space Lab.) program through the National Research Foundation of Korea funded by the Ministry of Education, Science and Technology (Grant 20090091724), and Ministry of Land, Transport and Maritime Affairs (MLTM) through the Super Long Span Bridge R\&D Center in Korea. 
Additional support from the Institute of Advanced Aerospace Technology at Seoul National University is also appreciated.

\section{REFERENCES}

1. Han, S,. Choi, H., Kim, C., Hwang, C., Jung, G. and Oh, J., “ALE-Based FSI Simulation of Solid Propellant Rocket Interior", 48th AIAA Aerospace Sciences Meeting Including the New Horizons Forum and Aerospace Exposition, AIAA, AIAA-2010-52, Orlando, Florida., January 2010

2. Kim, K. H., Kim, C., Rho, O.-H.,"Methods for Accurate Computations of Hypersonic flows, PART I : AUSMPW+ scheme", J. Comput. Phys. 178, pp.38 80, 2001

3. Rebay, S., "Efficient unstructured mesh generation by means of Delaunay triangulation and Boyer-Watson algorithm", J. Comput. Phys., vol. 106, pp.125-138, 1993

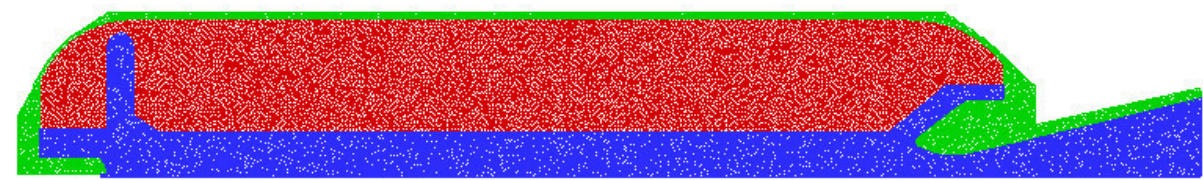

Figure. 1 Geometry of the solid rocket model used in simulation
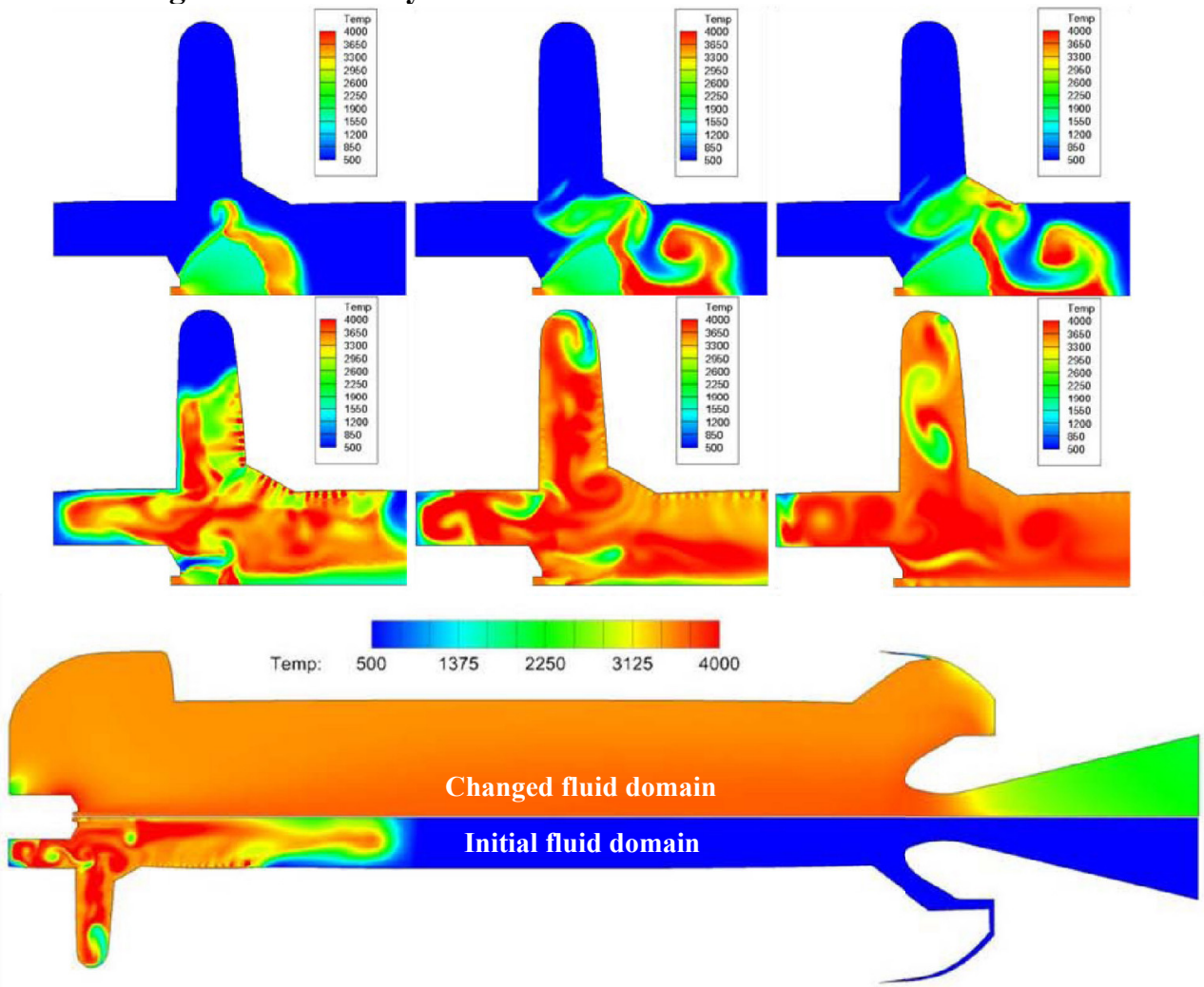

Figure. 2 Fluid results the solid rocket interior integrated simulation (top) Ignition process of solid propellant grain by pyrogen type igniter (bottom) Deformation of fluidic domain due to consumption of solid propellant 